\title{
Exemplification Modeling: Can You Give Me an Example, Please?
}

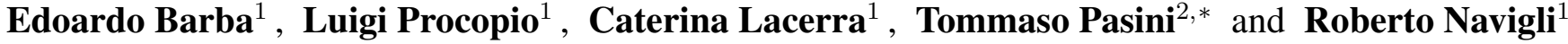 \\ ${ }^{1}$ Sapienza NLP Group, Department of Computer Science, Sapienza University of Rome \\ ${ }^{2}$ Department of Computer Science, University of Copenhagen \\ \{edoardo.barba, luigi.procopio, caterina.lacerra, roberto.navigli\}@ uniroma1.it \\ tommaso.pasini@di.ku.dk
}

\begin{abstract}
Recently, generative approaches have been used effectively to provide definitions of words in their context. However, the opposite, i.e., generating a usage example given one or more words along with their definitions, has not yet been investigated. In this work, we introduce the novel task of Exemplification Modeling (EXMOD), along with a sequenceto-sequence architecture and a training procedure for it. Starting from a set of (word, definition) pairs, our approach is capable of automatically generating high-quality sentences which express the requested semantics. As a result, we can drive the creation of sense-tagged data which cover the full range of meanings in any inventory of interest, and their interactions within sentences. Human annotators agree that the sentences generated are as fluent and semantically-coherent with the input definitions as the sentences in manually-annotated corpora. Indeed, when employed as training data for Word Sense Disambiguation, our examples enable the current state of the art to be outperformed, and higher results to be achieved than when using gold-standard datasets only. We release the pretrained model, the datasets and the software at https://github.com/SapienzaNLP/exmod.
\end{abstract}

\section{Introduction}

Providing the sense of a word given its context is a major topic in lexical semantics that has drawn considerable attention [Blevins and Zettlemoyer, 2020; Bevilacqua and Navigli, 2020; Barba et al., 2021]. Through the years, this task has mainly been formulated in two flavors: Word Sense Disambiguation [Bevilacqua et al., 2021, WSD], where the sense has to be chosen from a predefined sense inventory, and, more recently, Definition Modeling, where the sense definition for a word in context is automatically generated [Bevilacqua et al., 2020]. In particular, Definition Modeling drops the need for enumerative sense inventories and leverages instead the power of auto-regressive pretrained models to generate glosses, i.e., definitions, for arbitrary words and expressions.

\footnotetext{
${ }^{*}$ Work carried out while at the Sapienza University of Rome.
}

We claim that the reverse process, i.e., providing examples for a sense starting from its definition, has advantageous applications too, both for humans and automatic systems. Indeed, on the one hand, using examples to further describe word meanings can be beneficial for second language learners [Nation, 2001]. On the other hand, generated examples can be used to enrich pre-existing knowledge bases and as a data augmentation technique for Word Sense Disambiguation systems.

In this paper, we introduce the Exemplification Modeling (EXMOD) task, which aims at generating example sentences starting from one or multiple words with their sense definitions, together with a sequence-to-sequence architecture for the task, and a procedure for training it from sense-annotated corpora. We show that our model can generalize not only over unseen words and definitions, but also across different lexical resources with diverse sense granularities. Furthermore, the proposed architecture can provide examples for up to three senses at the same time, paving the way to new scenarios on how a generative system can model the interaction among multiple senses. We evaluate the produced sentences quantitatively by employing them as additional training examples for the WSD task. The examples generated lead WSD models to perform better than when relying on manually-annotated datasets only, and to attain results higher than the current state of the art. Furthermore, we analyze the sentences generated by means of a human-annotation task, and show that annotators perceive the examples produced as fluent and semantically coherent as those in a manually-curated corpus. This work brings the following novel contributions:

- The Exemplification Modeling (EXMOD) problem, i.e., an innovative task requiring systems to generate a usage example given one or more words with their sense definitions.

- A sequence-to-sequence architecture for ExMOD that can be trained directly on already-existing lexicalsemantic resources.

- An effective procedure for creating large-scale and highquality training corpora for English WSD covering all senses of the reference inventory.

- An in vivo evaluation framework taking into account different sense inventories and gold training corpora, as well as a human-evaluation task for measuring the fluency and semantic coherence of the generated sentences. 


\section{Methodology}

In this Section, we introduce and formalize the task of Exemplification Modeling (Section 2.1), describe our autoregressive approach for it (Section 2.2) and present the sampling strategy over the training instances (Section 2.3).

\subsection{Exemplification Modeling}

Given a set of lemmas along with their definitions, we frame Exemplification Modeling as the task of generating a sentence where each input lemma is used with its intended sense. ${ }^{1}$ For example, consider as input the lemma-definition pair (bank, a building where financial services are offered), a possible correct output might be: He went to the bank to deposit a check. Formally, let $D$ be the set of lemma-definition pairs $\left(l_{1}, d_{1}\right), \ldots,\left(l_{k}, d_{k}\right)$, with each definition $d_{i}$ specifying the intended meaning of lemma $l_{i}$; both $l_{i}$ and $d_{i}$ are sequences of tokens ${ }^{2}$ and we use $l_{i}^{j}$ and $d_{i}^{j}$ to denote their respective $j$-th token. The task can thus be formulated as follows: given the set $D$ of lemmadefinition pairs, a model has to yield a meaningful and semantically-coherent token sequence $s=s_{1}, \ldots, s_{n}$, such that, $\forall\left(l_{i}, d_{i}\right) \in D, l_{i}$ occurs in any of its inflected forms in $s$ with the meaning defined by its corresponding definition $d_{i}$. Furthermore, as knowing precisely where each $l_{i}$ occurs in $s$ represents a natural desideratum, we include as part of the task also yielding the set of indices $\phi_{i}=j_{i}^{1}, \ldots, j_{i}^{\left|\phi_{i}\right|}$ $\left(1 \leq j_{i}^{1}<\cdots<j_{i}^{\left|\phi_{i}\right|} \leq n\right)$ where $l_{i}$ is expressed in $s$.

\subsection{Model}

To tackle the ExMOD task, we propose a two-stage approach, which first generates a usage example $s$ where all the input lemmas occur with their expected meanings, and then computes $\phi_{i} \forall\left(l_{i}, d_{i}\right) \in D$ by means of a post-processing strategy. For the first stage, we use a sequence-to-sequence model and define its input $m$ as follows:

$$
\begin{aligned}
m= & <s>l_{1}^{1} \ldots l_{1}^{\left|l_{1}\right|}</ s>d_{1}^{1} \ldots d_{1}^{\left|d_{1}\right|} \\
& \ldots \\
& <s>l_{k}^{1} \ldots l_{k}^{\left|l_{k}\right|}</ s>d_{k}^{1} \ldots d_{k}^{\left|d_{k}\right|}
\end{aligned}
$$

where $\langle s>$ and $\langle/ s\rangle$ are two special tokens around which each input lemma is wrapped. The target example $s$ is similarly encoded into a sequence $\hat{s}$, where, $\forall\left(l_{i}, d_{i}\right) \in$ $D$, all contiguous spans in $\phi_{i}$ are surrounded by the special markers $\langle t\rangle$ and $\langle/ t\rangle$. For instance, given the above usage example He went to the bank to deposit a check, we convert it into He went to the $\langle t>$ bank $</ t>$ to deposit a check. With this encoding, we then train the sequence-to-sequence model to learn the factorized probability:

$$
p(\hat{s} \mid m)=\prod_{j=2}^{|\hat{s}|} p\left(\hat{s}_{j} \mid \hat{s}_{1: j-1}, m\right)
$$

\footnotetext{
${ }^{1}$ Lemma-definition pairs identify a sense for the lemma, therefore, we use "lemma-definition pair" and "sense" interchangeably.

${ }^{2}$ We model lemmas through lists of tokens to support multiwords.
}

by minimizing the cross-entropy loss with respect to $\hat{s}$.

However, as this formulation does not compute $\phi_{i} \forall\left(l_{i}, d_{i}\right) \in D$, we perform the second stage of our approach and, denoting with $o$ the sequence generated by our model, we apply the following four steps:

- We lemmatize each token in $o$;

- We pair each highlighted span, tagged with lemma $l$, to the pair $\left(l_{i}, d_{i}\right)$ such that $l_{i}=l ;{ }^{3}$

- We compute $\phi_{i}$ by considering the indices of the span aligned to each input pair $\left(l_{i}, d_{i}\right){ }^{4}$

- We remove the highlighting markers from the sequence. After these four steps, the system produces a usage example $s$ for the input pairs in $D$, along with a set of indices $\phi_{i}$ indicating where the $i$-th input pair is expressed within $s$.

\subsection{Sampling}

We now present the method we use for sampling the training instances from a given collection $B=$ $\left(D_{1}, s_{1}\right), \ldots,\left(D_{|B|}, s_{|B|}\right)$ of inputs and expected outputs. In this work, we limit ourselves to consider only $k=\left|D_{i}\right| \in$ $\{1,2\}$ for $i=1, \ldots,|B|$ and defer exploring training strategies with $k \geq 3$ for future work. We believe that these two values of $k$ are significantly different from each other, as sense interactions enter the picture when dealing with $k=2$, and we further argue that their modeling calls for different approaches. For $k=1$, we assume all instances in $B$ be equally adequate and define a uniform distribution over this list, sampling $\left(D_{i}, s_{i}\right)$ with probability:

$$
p\left(D_{i}, s_{i}\right)=\frac{1}{|B|}
$$

For $k=2$, instead, we focus on usage examples where the senses in $D_{i}$ appear as lexical collocations ${ }^{5}$ of one another and, thus, employ a $p\left(D_{i}, s_{i}\right)$ that fosters this choice. That is, for each input $D_{i}=\left\{\left(l_{i, 1}, d_{i, 1}\right),\left(l_{i, 2}, d_{i, 2}\right)\right\}$ and its target sentence $s_{i}$, we set its probability $p\left(D_{i}, s_{i}\right)$ to 0 if the senses in $D_{i}$ appear more than $\xi$ tokens away from each other in $s_{i}$. For all the remaining instances $\hat{B}$, instead, we compute their probability based on the Positive Pointwise Mutual Information $^{6}$ (PPMI, Niwa and Nitta [1994]) measure:

$$
p\left(D_{i}, s_{i}\right)=(1-\alpha) \frac{p p m i\left(D_{i}\right)}{\sum_{j=1}^{|\hat{B}|} p p m i\left(D_{j}\right)}+\alpha \frac{1}{\sigma\left(s_{i}\right)|S|}
$$

where $\sigma\left(s_{i}\right)$ is the number of $\left(D_{j}, s_{j}\right)$ pairs such that $s_{j}=$ $s_{i}, S$ is the set of all the usage examples, and ppmi is the function defined as:

$$
\operatorname{ppmi}\left(D_{i}\right)=\max \left(0, \log \frac{p\left(l_{i, 1}, l_{i, 2}\right)}{p\left(l_{i, 1}\right) p\left(l_{i, 2}\right)}\right)
$$

\footnotetext{
${ }^{3}$ If multiple pairs match $l$, we assign it to one at random.

${ }^{4}$ If no input pair is associated with any span, we discard the example.

${ }^{5}$ Pairs of words that co-occur more frequently than chance.

${ }^{6}$ We estimate the probability of the occurrence of a given word by counting its occurrences in the English Wikipedia corpus (December 2019 dump). Further details at https://github.com/SapienzaNLP/ exmod (D).
} 
The second term of Equation 1 is effectively a smoothing factor $^{7}$ that distributes a uniform probability over each usage example $s_{i}$, dividing it equally among all the training instances in which it occurs. With this strategy, we effectively foster the sampling of $\left(D_{i}, s_{i}\right)$ pairs such that:

- $\left(l_{i, 1}, d_{i, 1}\right)$ and $\left(l_{i, 2}, d_{i, 2}\right)$ co-occur nearby;

- the co-occurrence is statistically significant.

Therefore, we encourage the generation of usage examples where the input lemmas actively interact between each other.

\section{In Vivo Evaluation}

In this Section, we put forward an in vivo evaluation suite for the Exemplification Modeling task. To this end, we focus on the Word Sense Disambiguation (WSD) problem, which, given a word in context, aims at selecting its most suitable meaning, and design 5 sub-tasks to evaluate different aspects of the examples generated by an ExMOD model. Across all sub-tasks, we make use of the following lexical resources:

- WordNet [Miller et al., 1990], an electronic dictionary where textual definitions, i.e., glosses, are assigned to synsets, lexical units denoting a group of synonymous senses, each associated with a different lemma.

- SyntagNet [Maru et al., 2019], a manually-curated resource of semantic collocations, i.e., pairs of WordNet senses that appear frequently together in texts.

- SemCor [Miller et al., 1993], a large sense-annotated corpus for WSD, comprising $33 \mathrm{~K}$ sentences for $200 \mathrm{~K}$ instances tagged with WordNet senses.

- Princeton WordNet Gloss Corpus (WNG) ${ }^{8}$, a corpus consisting of tagged examples and definitions in WordNet. We keep only the examples and the resulting dataset features 34,275 instances. ${ }^{9}$

- The Oxford Dictionary Dataset [Chang et al., 2018], containing 555,695 sentences for as many instances tagged with senses from the Oxford Dictionary of English (ODE).

\subsection{Tasks}

For each task, we define the data that the ExMOD system will use during the training and generation phases. We also report the cardinality $k$ of the input set of lemma-definition pairs and the sense inventory from which the input pairs have to be drawn. In what follows, we delineate the five WSD sub-tasks:

Task 1 In this sub-task, we test the capabilities of EXMOD models to generate data in the simplest scenario, that is, producing examples for a single sense at a time from the same sense inventory that was used during the training phase.

Training: We set $k=1$, therefore serving only $(D, s)$ pairs with $|D|=1$, and draw the training pairs from the concatenation of SemCor and WNG.

Generation: We query the model with all lemma-definition pairs contained in WordNet and build the WSD dataset from the resulting examples.

\footnotetext{
${ }^{7}$ We use $\alpha=0.15$.

${ }^{8} \mathrm{https}: / /$ wordnetcode.princeton.edu/glosstag.shtml

${ }^{9}$ Please refer to https://github.com/SapienzaNLP/exmod (A).
}

Task 2 This task is analogous to the previous one as far as $k$ is concerned, but differs in the inventories used at training time. This setting, indeed, aims at assessing the capability of a model to perform zero-shot generations for senses coming from a previously unseen inventory.

Training: We set $k=1$ and select the training instances from the Oxford Dictionary Dataset.

Generation: We query the model analogously to Task 1 and build the WSD dataset from the resulting examples.

Task 3 The third task challenges ExMOD models to generate examples that are coherent with two input senses.

Training: We vary $k \in\{1,2\}$, therefore serving training pairs $(D, s)$ with $|D| \leq 2$. To build the set of training instances when $k=1$, we consider all WordNet senses appearing in the concatenation of SemCor and WNG. As for $k=2$, we collect all the sense pairs appearing in a SemCor sentence and discard all those whose senses are further away than a window of $\xi=10$ tokens. We recall from Section 2.3 that we sample each pair according to Equation 1.

Generation: We query the model with all the possible pairs of senses $(k=2)$ in SemCor appearing within a window of size 10, and all sense pairs of SyntagNet.

Task 4 This task evaluates the generalization ability of an ExMOD model to scale from inputs with $k \leq 2$ used at training time, to inputs with $k=3$ used at generation time.

Training: We keep the same training setting as Task 3 .

Generation: We query the model to generate examples for triplets of senses $(k=3)$ that we compute by enumerating all sense triangles in SyntagNet, i.e., sets of three senses, each in collocation with the other two.

Task 5 The last task aims at pushing an ExMOD model to its limit by considering all the possible resources at training and generation time.

Training: We vary $k \in\{1,2\}$. For $k=1$, we extract the training instances by considering all WordNet senses appearing in SemCor or WNG and all ODE senses in the Oxford Dictionary Dataset. For $k=2$, we use the setting of Task 3 . Generation: We query the model varying $k \in\{1,2,3\}$ and draw i) senses from WordNet, ii) pairs from SemCor and SyntagNet, and iii) triplets from SyntagNet triangles.

\subsection{Setup}

In this Section, we detail the ExMOD and WSD models used for the experiments, as well as their training hyperparameters, the generation strategy and the evaluation data. Finally, we also propose a metric for model selection that takes into account the semantic coherence of the sentence produced with respect to the input senses.

ExMoD Model and Training. We use BART [Lewis et $a l ., 2020]$ as the underlying sequence-to-sequence architecture of our approach; henceforth, we refer to our model as EXMAKER. We train EXMAKER with RAdam [Liu et al., 2020] for 300,000 training steps, learning rate set to $1 e^{-5}$ and batches of 800 tokens, accumulating gradient for 10 steps. When dealing with multiple datasets, we perform batch sampling from each of them. 
ExMoD Model Selection. Choosing the set of weights of EXMAKER that yields the best generations is not a trivial task. Indeed, as multiple usage examples may be perfectly adequate for a given set $D$, our generative approach is an instance of open-ended text generation where referenced metrics, such as the cross-entropy loss over a reserved sample of data, have been shown to be unsuitable [Liu et al., 2016]. To overcome this issue, we propose an unreferenced metric for measuring the semantic coherence of the example generated with respect to its input set $D$. Specifically, we take advantage of ARES [Scarlini et al., 2020], i.e., latent representations of WordNet senses that lie in a space comparable to that of BERT Large [Devlin et al., 2019]. Given a generated sentence and a target word therein, we extract the target word embedding by means of BERT Large and compute its cosine similarity with the ARES embedding of the input sense. Thus, to perform model selection during training, we define two sets of validation instances for $k=1$ and $k=2$, with 300 and 580 samples, respectively, ${ }^{10}$ and calculate this similarity for each pair in each input set $D$, aggregating them via a macro average. We compute the ARES-score every 2000 training steps and select the model with the highest performance in terms of macro average.

Generation. In each subtask, we generate from 1 to 6 examples given a set of lemma-definition pairs $D$ by applying the following decoding strategies in parallel on EXMAKER:

- beam-nl: beam search returning the best beam;

- sample-n5: nucleus-sampling with $p=0.9$ returning up to 5 sequences.

We group the outputs and clean them by discarding all the ill-formed generations, that is, we discard all those examples generated from $D$ such that $\forall\left(l_{i}, d_{i}\right) \in D, \phi_{i}=\emptyset$. Finally, using $\phi_{i}$, we tag the highlighted tokens with the sense in the reference inventory identified by the $\left(l_{i}, d_{i}\right)$ pair.

WSD Reference Model. As reference WSD system, we choose a simple yet effective Transformer-based solution, i.e., BERT for token classification. Following Devlin et al. [2019], we represent each token ${ }^{11}$ through the concatenation of the last four layers of BERT, and apply a linear classification head to map each token to a sense. We train the model for at most 50 epochs with early stopping on the validation accuracy and patience set to 3 epochs. ${ }^{12}$ As training data, we use the concatenation of SemCor and WNG together with the silver data generated for each specific experiment.

Comparison Systems. As comparison, we report the results of the reference system trained on SemCor and WNG only, as well as on their concatenation with two other automatically-produced datasets, i.e., OneSeC [Scarlini et al., 2019], which relies on information within a knowledge base to tag Wikipedia sentences, and OMSTI [Taghipour and Ng, 2015], a semi-automatic approach relying on parallel corpora.

\footnotetext{
${ }^{10}$ Please refer to https://github.com/SapienzaNLP/exmod (B.1) for more details on their creation.

${ }^{11}$ We use the first sub-word embedding when the token is split.

${ }^{12}$ Please refer to https://github.com/SapienzaNLP/exmod (C) for all hyperparameters.
}

To put our results in context with the state of the art, we also report the result of the currently best-performing system, i.e., ESCHER [Barba et al., 2021]. We report its results when trained on i) SemCor, ii) SemCor, WNG and the Oxford Dictionary Dataset, and iii) SemCor, WNG and EXMAKER data.

WSD Evaluation Data. As standard in WSD, we use the evaluation framework made available by Raganato et al. [2017]. The framework contains six test datasets, namely, Senseval-2 [Palmer et al., 2001], Senseval-3 [Snyder and Palmer, 2004], SemEval-07 [Pradhan et al., 2007], SemEval13 [Navigli et al., 2013] and SemEval-15 [Moro and Navigli, 2015]; and ALL, the concatenation of all the aforementioned datasets. To examine the models' ability to generalize over rare and unseen senses, we partition the ALL dataset into the three following splits:

- MFS, containing all instances tagged with the Most Frequent Sense (MFS) in SemCor and WNG for a specific lemma.

- LFS, containing all the instances that are tagged with a Least Frequent Sense, i.e., a sense that is not the MFS.

- Unseen, containing all the instances tagged with a sense that never appears either in SemCor or in WNG.

\subsection{Results}

In Table 1, we report the results of our reference model in each sub-task, as well as the various baselines.

We first compare the reference model when trained on SemCor with the WNG Examples only and when trained with additional data for the WordNet inventory coming from OMSTI, OneSeC and ExMAKER (Task 1). As one can see, the data generated by EXMAKER boosts BERT performance by 1.3 points, a result that none of the other additional datasets achieves. More interestingly, the improvement comes entirely from senses that are underrepresented in the training set (LFS and Unseen columns). These results highlight the unique ability of EXMAKER to produce examples for senses that are either rarely or never seen in the gold datasets, while confirming that a model trained for the EXMOD task can be employed effectively to generate examples for WordNet senses.

By considering the results in the second task (Task 2), we can state with confidence that EXMAKER, while being trained with definitions from a single inventory (ODE), can provide examples for senses in another inventory (WordNet) that has different sense granularity. Furthermore, training on instances from EXMAKER in Task 2 results in higher WSD performance than when training on the sentences generated by EXMAKER in Task 1. This result paves the way for new scenarios where ExMOD models are used to complete existing knowledge bases, e.g., WordNet, which lacks examples for roughly $85 \%$ of its senses. ${ }^{13}$

We now focus on results for Task 3 and 4, which test the ability of EXMAKER to create coherent examples for pairs or triplets of senses when trained on single definitions or pairs of definitions. Results show that the sentences generated with $k=2$ are beneficial to the WSD model and further boost its

\footnotetext{
${ }^{13} 207,016$ in total.
} 


\begin{tabular}{|c|c|c|c|c|c|c|c|c|c|}
\hline & \multirow[b]{2}{*}{ Task } & \multicolumn{3}{|c|}{ WSD Setting } & \multirow{2}{*}{$\frac{\text { Dev Set }}{\text { SE7 }}$} & \multicolumn{4}{|c|}{ Test Sets } \\
\hline & & Model & Additional Dataset & $\mathrm{k}$ & & MFS & LFS & Unseen & ALL \\
\hline \multirow{5}{*}{ 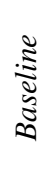 } & - & BERT & - & - & 69.6 & 95.1 & 42.5 & 53.43 & 74.4 \\
\hline & - & BERT & OMSTI & - & 68.4 & 93.7 & 45.5 & 53.5 & 74.5 \\
\hline & - & BERT & OneSec & - & 67.3 & 95.3 & 39.5 & 54.0 & 73.9 \\
\hline & - & $\mathrm{ESCHER}^{\dagger}$ & - & - & 76.3 & 93.7 & 55.7 & 75.0 & 80.7 \\
\hline & - & ESCHER & ODE & - & 77.9 & 94.6 & 56.4 & 76.8 & $\underline{81.6}$ \\
\hline \multirow{6}{*}{ 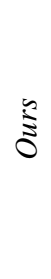 } & Task 1) & BERT & EXMAKER & 1 & 69.8 & 93.2 & 48.1 & 58.9 & 75.7 \\
\hline & Task 2) & BERT & EXMAKER & 1 & 70.1 & 93.3 & 48.3 & 59.1 & 76.0 \\
\hline & Task 3) & BERT & EXMAKER & 2 & 69.9 & 93.0 & 48.4 & 59.8 & 76.2 \\
\hline & Task 4) & BERT & EXMAKER & 3 & 70.0 & 93.1 & 48.0 & 60.3 & 76.1 \\
\hline & Task 5) & BERT & EXMAKER & $1,2,3$ & 71.2 & 95.7 & 47.3 & 57.8 & 76.8 \\
\hline & Task 5) & ESCHER & EXMAKER & $1,2,3$ & 78.1 & 94.8 & 58.6 & 77.1 & $\underline{82.3}$ \\
\hline
\end{tabular}

Table 1: F1 attained when training the reference model, BERT, on the gold training set (the concatenation of SemCor and WNG) concatenated with several silver datasets generated by different configurations of EXMAKER and its competitors, i.e., OMSTI and OneSeC. $\dagger$ indicates that the model is trained on SemCor instances only. Underlined scores are statistically significant with respect to ESCHER results with $p<0.05$.

performance from 75.7 (Task 1) to 76.2 (Task 3). We hypothesize that this result is a consequence of the more complete semantics provided to EXMAKER, enabling it to generate sentences where the senses occur with a sharper connotation, thus providing, in turn, a clearer context to a WSD model. Considering the dataset generated with $k=3$ (Task 4), the WSD model performance is comparable to that attained when trained on a dataset generated with $k=2$ (Task 3). As EXMAKER is not trained to generate examples for triplets of word-definitions pairs, this surprising result highlights its capability to produce coherent sentences even for sets of senses larger than those seen during training.

Finally, we discuss the last task, where we aim at measuring the maximum performance that a WSD model can reach when using data from EXMAKER, i.e., when trained on all resources and used for generating sentences with $k \in$ $\{1,2,3\}$. In this setting we also train the state-of-the-art model for WSD (ESCHER) on the concatenation of SemCor, WNG and EXMAKER data. Thanks to EXMAKER data, ESCHER reaches an unprecedented result of 82.3 points of $\mathrm{F} 1$, performing better than when using manually-annotated data only (SemCor, WNG Examples and the Oxford Dictionary Dataset), and surpassing the current state of the art (80.7) by 1.6 points. We also note that BERT performance gains 0.5 and 0.6 points on ALL with respect to Tasks 3 and 4, resulting in an overall improvement of 1.1 points over Task 1.

Through these quantitative experiments, we show that EXMAKER produces high-quality sentences even when exemplifying rare and unseen senses, fully taking advantage of different resources and sense inventories. Furthermore, the datasets generated prove to be useful as additional resources for WSD models, leading them to set a new state of the art.

\section{Qualitative Analysis}

We now focus on qualitatively evaluating the EXMAKER examples by means of two annotation tasks. We ask annotators to manually assign two scores to each example generated: one

\begin{tabular}{lccllc}
\hline \multirow{2}{*}{ Task } & \multicolumn{2}{c}{ ALL } & & \multicolumn{2}{c}{ ExMAKER } \\
\cline { 2 - 3 } \cline { 5 - 6 } & AVG & $\kappa$ & & AVG & $\kappa$ \\
\hline Fluency & 4.82 & 0.74 & & 4.63 & 0.70 \\
Coherence & 4.83 & 0.69 & & 4.82 & 0.68 \\
\hline
\end{tabular}

Table 2: Results for the two tasks of qualitative analysis. We report the Likert scores average and the pairwise average of Cohen's $\kappa$.

measuring its fluency and one measuring its semantic coherence with respect to a given lemma-definition pair.

Data to Annotate. We build two datasets: one automatically generated by EXMAKER trained as in Task 5, and one drawn from the ALL dataset. We consider a statistically significant sample ${ }^{14}$ of 284 and 382 instances of the ALL and EXMAKER datasets, respectively, shuffle them together, and provide the anonymized examples to three English-proficient annotators. ${ }^{15}$

Annotation Task. We provide annotators with guidelines ${ }^{16}$ and ask them to tag each example with two scores: the first, to evaluate its fluency and indicate whether it is logical and grammatically correct; the second, to measure to what extent the usage of the target word in the example reflects the meaning described by the given definition. Following Bevilacqua et al. [2020], in both cases, we ask the annotators to fill a five-level Likert scale that assigns higher scores to better sentences. We report the average computed across all instances and annotators for each measure and Cohen's $\kappa$ [Cohen, 1960] as metric for the Inter-Annotator Agreement.

Results. As one can see in Table 2, the average Likert scores for EXMAKER data are 4.63 and 4.82 for fluency and semantic coherence, respectively. These results are nearly

\footnotetext{
${ }^{14}$ Samples are statistically significant w.r.t. the sizes of the source datasets with confidence level of $95 \%$ and a margin error of \pm 5 .

${ }^{15}$ Annotators do not know the source of each sentence.

${ }^{16}$ Released at https://github.com/SapienzaNLP/exmod (E).
} 
identical to those attained for the manually-annotated examples of ALL. The inter-annotator agreement is substantial [Landis and Koch, 1977] for both datasets and measures. These results underline the high quality of the sentences produced by EXMAKER, which not only have a fluency close to that of human-curated corpora, but are also capable of conveying the required input meaning into valid examples that turn out to be only slightly worse on average than those created by humans.

\section{Related Work}

Definition Modeling The spread of generative models is fostering the advancement of research in several topics, among which definition modeling [Noraset et al., 2017] is the closest to ours. The task requires a gloss to be produced for a word, and was initially proposed as an interpretable way for analyzing the semantics of word embeddings. Early approaches to the task [Gadetsky et al., 2018; Chang et al., 2018] relied mainly on static word embeddings to model the input context and generate the most suitable definition for the target word. Static word embeddings were then replaced by contextualized embeddings, better modeling the semantics of a target word in context. Nevertheless, these approaches were neither able to provide definitions for multiword expressions [Mickus et al., 2019], nor to take into account the word order when defining multiword expressions [Ishiwatari et al., 2019]. The most recent effort in this direction is Generationary [Bevilacqua et al., 2020], which exploits a sequence-to-sequence generative approach where the spans to be defined are explicitly marked. This approach closed the gap between Definition Modeling and Word Sense Disambiguation, showing that the glosses generated could easily be mapped to those in a lexical knowledge base, hence making it possible to link the target span to a sense in a dictionary.

Word Sense Disambiguation Differently from Definition Modeling, Word Sense Disambiguation is a long-standing task in NLP [Navigli, 2009], which aims at assigning each content word in a text to its most suitable meaning drawn from a sense inventory. Approaches to this task are either knowledge-based, such as graph and heuristic-driven algorithms [Maru et al., 2019], or supervised, such as neural networks [Bevilacqua and Navigli, 2020; Blevins and Zettlemoyer, 2020]. Knowledge-based methods rely solely on lexical-semantic knowledge bases, and, in general, perform worse than their supervised counterparts. These latter, indeed, consistently attain state-of-the-art results thanks to their ability to learn from data. Nevertheless, their results are still limited by the lack of large-scale sense-annotated corpora: currently-used datasets cover less than $20 \%$ of the senses in WordNet, i.e., the de facto standard sense inventory of English.

Automatically-generated Data for WSD Since supervised approaches show better performance in general, several efforts have been put into creating sense-annotated corpora automatically. OMSTI [Taghipour and $\mathrm{Ng}, 2015$ ] exploited human annotations of Chinese senses within a parallel corpus to automatically disambiguate their corresponding English sentences. MuLaN [Barba et al., 2020] also employed human annotations, but focused on producing data in languages other than English by relying on a cross-lingual sentence retrieval step to project English annotations potentially to hundreds of languages. Conversely, OneSeC [Scarlini et al., 2019] does not require manually-annotated data, but instead leverages the information within a knowledge base and in Wikipedia to produce sense-tagged data.

To the best of our knowledge, this work is the first to formulate the Exemplification Modeling (ExMOD) task, i.e., the task of generating example sentences given one or more sense definitions. ExMOD is similar in its generative nature to Definition Modeling, however, its goal is to provide usage examples of selected word meanings, rather than defining words in contexts. As a by product, the proposed architecture for the task (EXMAKER) can generate data to train models for the Word Sense Disambiguation problem. Despite not being the main goal of this work, our approach is novel in comparison to other methods for creating silver data. Indeed, EXMAKER generates examples ex novo and on demand, while all the other systems tag already-existing sentences.

\section{Conclusions}

In this work, we introduced the new task of Exemplification Modeling (EXMOD), aimed at generating a usage example for a given set of words with their definitions. We showed that the task can be tackled by means of an encoder-decoder architecture (EXMAKER) trained on already-available data for Word Sense Disambiguation (WSD). Human evaluation showed that the examples produced can be confused with those drawn from a manually-curated corpus as they are fluent and semantically-coherent with the input. Finally, we proposed an in vivo evaluation to measure the performance of systems for the EXMOD task automatically. This was based on the supervised WSD task, where the examples generated for the EXMOD task could be used to train a WSD model. Results show that the examples provided by EXMAKER lead WSD models to attain better performance than when using manually-tagged data only, while, at the same time, paving the way towards a full-fledged generative approach for data augmentation in Word Sense Disambiguation.

As future work we plan to expand the generation of examples on languages other than English and to enlarge the number of input senses that the architecture can handle. We release the software, all data and the annotation guidelines for the human-evaluation task at https://github.com/ SapienzaNLP/exmod.

\section{Acknowledgments}

The authors gratefully acknowledge the support of the ERC Consolidator Grant MOUSSE No. 726487 under the European Union's Horizon 2020 research and innovation programme.

This work was partially supported by the MIUR under the grant "Dipartimenti di eccellenza 2018-2022" of the Department of Computer Science of the Sapienza University of Rome. 


\section{References}

[Barba et al., 2020] Edoardo Barba, Luigi Procopio, Niccolò Campolungo, Tommaso Pasini, and Roberto Navigli. MuLaN: Multilingual Label propagatioN for Word Sense Disambiguation. In Proc. of IJCAI, 2020.

[Barba et al., 2021] Edoardo Barba, Tommaso Pasini, and Roberto Navigli. ESC: Redesigning WSD with extractive sense comprehension. In Proc. of NAACL, pages 4661-4672, 2021.

[Bevilacqua and Navigli, 2020] Michele Bevilacqua and Roberto Navigli. Breaking through the $80 \%$ glass ceiling: Raising the state of the art in word sense disambiguation by incorporating knowledge graph information. In Proc. of ACL, pages 28542864, 2020.

[Bevilacqua et al., 2020] Michele Bevilacqua, Marco Maru, and Roberto Navigli. Generationary or: "how we went beyond word sense inventories and learned to gloss". In Proc. of EMNLP, pages 7207-7221, 2020.

[Bevilacqua et al., 2021] Michele Bevilacqua, Tommaso Pasini, Alessandro Raganato, and Roberto Navigli. Recent trends in Word Sense Disambiguation: A survey. In Proc. of IJCAI, 2021.

[Blevins and Zettlemoyer, 2020] Terra Blevins and Luke Zettlemoyer. Moving down the long tail of word sense disambiguation with gloss informed bi-encoders. In Proc. of ACL, pages 10061017, 2020.

[Chang et al., 2018] Ting-Yun Chang, Ta-Chung Chi, Shang-Chi Tsai, and Yun-Nung Chen. xSense: Learning sense-separated sparse representations and textual definitions for explainable word sense networks. arXiv preprint arXiv:1809.03348, 2018.

[Cohen, 1960] Jacob Cohen. A coefficient of agreement for nominal scales. Educational and psychological measurement, 20(1):37-46, 1960.

[Devlin et al., 2019] Jacob Devlin, Ming-Wei Chang, Kenton Lee, and Kristina Toutanova. BERT: Pre-training of deep bidirectional transformers for language understanding. In Proc. of NAACL, pages 4171-4186, 2019.

[Gadetsky et al., 2018] Artyom Gadetsky, Ilya Yakubovskiy, and Dmitry Vetrov. Conditional generators of words definitions. In Proc. of ACL, pages 266-271, 2018.

[Ishiwatari et al., 2019] Shonosuke Ishiwatari, Hiroaki Hayashi, Naoki Yoshinaga, Graham Neubig, Shoetsu Sato, Masashi Toyoda, and Masaru Kitsuregawa. Learning to describe unknown phrases with local and global contexts. In Proc. of NAACL, pages 3467-3476, 2019.

[Landis and Koch, 1977] J Richard Landis and Gary G Koch. The measurement of observer agreement for categorical data. biometrics, pages 159-174, 1977.

[Lewis et al., 2020] Mike Lewis, Yinhan Liu, Naman Goyal, Marjan Ghazvininejad, Abdelrahman Mohamed, Omer Levy, Veselin Stoyanov, and Luke Zettlemoyer. BART: Denoising sequenceto-sequence pre-training for natural language generation, translation, and comprehension. In Proc. of ACL, pages 7871-7880, 2020.

[Liu et al., 2016] Chia-Wei Liu, Ryan Lowe, Iulian Serban, Mike Noseworthy, Laurent Charlin, and Joelle Pineau. How NOT to evaluate your dialogue system: An empirical study of unsupervised evaluation metrics for dialogue response generation. In Proc. of EMNLP, pages 2122-2132, 2016.

[Liu et al., 2020] Liyuan Liu, Haoming Jiang, Pengcheng $\mathrm{He}$, Weizhu Chen, Xiaodong Liu, Jianfeng Gao, and Jiawei Han. On the variance of the adaptive learning rate and beyond. In ICLR, 2020.

[Maru et al., 2019] Marco Maru, Federico Scozzafava, Federico Martelli, and Roberto Navigli. SyntagNet: Challenging supervised word sense disambiguation with lexical-semantic combinations. In Proc. of EMNLP, pages 3534-3540, 2019.

[Mickus et al., 2019] Timothee Mickus, Denis Paperno, and Matthieu Constant. Mark my word: A sequence-to-sequence approach to definition modeling. In Proc. of DeepNLP, 2019.

[Miller et al., 1990] George A. Miller, R.T. Beckwith, Christiane D. Fellbaum, D. Gross, and K. Miller. Introduction to WordNet: an online lexical database. International Journal of Lexicography, 3(4):235-244, 1990.

[Miller et al., 1993] George A Miller, Claudia Leacock, Randee Tengi, and Ross T Bunker. A semantic concordance. In Proc. of Human Language Technology, pages 303-308, 1993.

[Moro and Navigli, 2015] Andrea Moro and Roberto Navigli. SemEval-2015 task 13: Multilingual all-words sense disambiguation and entity linking. In Proc. of SemEval, pages 288 297, 2015.

[Nation, 2001] Ian SP Nation. Learning vocabulary in another language. Cambridge University Press, 2001.

[Navigli et al., 2013] Roberto Navigli, David Jurgens, and Daniele Vannella. SemEval-2013 task 12: Multilingual word sense disambiguation. In Proc. of SemEval, pages 222-231, 2013.

[Navigli, 2009] Roberto Navigli. Word Sense Disambiguation: A survey. ACM computing surveys (CSUR), 41(2):1-69, 2009.

[Niwa and Nitta, 1994] Yoshiki Niwa and Yoshihiko Nitta. Cooccurrence vectors from corpora vs. distance vectors from dictionaries. In Proc. of COLING, 1994.

[Noraset et al., 2017] Thanapon Noraset, Chen Liang, Larry Birnbaum, and Doug Downey. Definition modeling: Learning to define word embeddings in natural language. In Proc. of AAAI, 2017.

[Palmer et al., 2001] Martha Palmer, Christiane Fellbaum, Scott Cotton, Lauren Delfs, and Hoa Trang Dang. English tasks: Allwords and verb lexical sample. In Proc. of Senseval-2, pages 21-24, 2001.

[Pradhan et al., 2007] Sameer Pradhan, Edward Loper, Dmitriy Dligach, and Martha Palmer. SemEval-2007 task-17: English lexical sample, SRL and all words. In Proc. of SemEval, pages 87-92, 2007.

[Raganato et al., 2017] Alessandro Raganato, Jose CamachoCollados, and Roberto Navigli. Word Sense Disambiguation: A unified evaluation framework and empirical comparison. In Proc. of EACL, pages 99-110, 2017.

[Scarlini et al., 2019] Bianca Scarlini, Tommaso Pasini, and Roberto Navigli. Just "OneSeC" for producing multilingual sense-annotated data. In Proc. of ACL, pages 699-709, 2019.

[Scarlini et al., 2020] Bianca Scarlini, Tommaso Pasini, and Roberto Navigli. With more contexts comes better performance: Contextualized sense embeddings for all-round word sense disambiguation. In Proc. of EMNLP, pages 3528-3539, 2020.

[Snyder and Palmer, 2004] Benjamin Snyder and Martha Palmer. The English all-words task. In Proc. of Senseval-3, pages 4143, 2004.

[Taghipour and Ng, 2015] Kaveh Taghipour and Hwee Tou Ng. One million sense-tagged instances for word sense disambiguation and induction. In Proc. of CoNLL, pages 338-344, 2015. 